\title{
Observing quantum non-locality in the entanglement between modes of massive particles
}

\author{
S. Ashhab, ${ }^{1}$ Koji Maruyama, ${ }^{1,2}$ and Franco Nori ${ }^{1,3}$ \\ ${ }^{1}$ Frontier Research System, The Institute of Physical and Chemical Research (RIKEN), Wako-shi, Saitama 351-0198, Japan \\ ${ }^{2}$ Laboratoire d'Information Quantique and QUIC, CP 165/59, \\ Université Libre de Bruxelles, 1050 Bruxelles, Belgium \\ ${ }^{3}$ Physics Department and Michigan Center for Theoretical Physics, \\ The University of Michigan, Ann Arbor, Michigan 48109-1040, USA
}

(Dated: August 1, 2018)

\begin{abstract}
We consider the question of whether it is possible to use the entanglement between spatially separated modes of massive particles to observe nonlocal quantum correlations. Mode entanglement can be obtained using a single particle, indicating that it requires careful consideration before concluding whether experimental observation, e.g. violation of Bell inequalities, is possible or not. In the simplest setups analogous to optics experiments, that observation is prohibited by fundamental conservation laws. However, we show that using auxiliary particles, mode entanglement can be converted into forms that allow the observation of quantum non-locality. The probability of successful conversion depends on the nature and number of auxiliary particles used. In particular, we find that an auxiliary Bose-Einstein condensate allows the conversion arbitrarily many times with a small error that depends only on the initial state of the condensate.
\end{abstract}

\section{INTRODUCTION}

Entanglement has attracted interest ever since it was recognized as a central ingredient in quantum mechanics [1], both on a fundamental level as it represents a clear deviation from classical intuition [2] and, recently, for its possible use as a resource in the emerging field of quantum information processing [3].

One typically thinks of entanglement as describing quantum correlations between physical variables of two distinct particles or systems. Perhaps the simplest example is the Bell states of two (distinguishable) spin- $1 / 2$ particles. There are, however, a number of other situations where entanglement arises. For example, in the Stern-Gerlach experiment, the position of a particle is entangled with its spin. Another example, which will be the main focus of the present paper and will be explained shortly, is the so-called mode entanglement. On a more abstract level, one could even redefine a single physical degree of freedom (rather unnaturally) such that certain superpositions of basis states have the appearance of entangled states [4].

Mode entanglement can be perhaps most easily understood as follows: let us take a single particle in a quantum superposition of two basis states. In the field-theory description, we treat the modes (or, in other words, the basis states) as the quantum objects: each mode can be in the state of being occupied by zero particles, one particle and so on. Particles are therefore seen as excitations of the field modes. Going back to our example with a single particle in a superposition state, we find the alternative description where the two modes are in an entangled state: a quantum superposition where one mode is occupied and the other mode is empty, with the two possible combinations present in the superposition.
Given the wide range of manifestations of entanglement explained above, not all of them are equally intriguing. It is in fact the combination of entanglement and non-locality that fascinates us physicists the most. In particular, the entanglement between two degrees of freedom of a single object can be easily overlooked in this context because it does not contradict intuitive expectations about locality. One must therefore divide entanglement into two types: loosely speaking, one can speak of interesting versus uninteresting entanglement.

Clearly the above classification of the different types of entanglement according to how interesting they are is not unambiguous. One must therefore define that classification according to an unambiguous experimental procedure. It seems to us that the most reasonable definition would include the observation of nonlocal, non-classical correlations between two objects. The term 'nonlocal' is used in this context to mean the following: if we are to say that there is nonlocal entanglement (in an unquestionable form) between two objects at a given point in time, we require that quantum correlations be observable within a time $d / c$, where $d$ is the distance between the two objects, and $c$ is the speed of light [2, 5,6$]$.

An important requirement needed to detect entanglement is the ability to perform measurements in different bases. For example, if we take an ensemble of pairs of spin- $1 / 2$ particles and we find that the ensemble has perfect correlations (within each pair) in the measured values of the spins along the $z$-axis, we still cannot say for sure that the pairs are entangled. These observations would be consistent with classical correlations. Only after performing measurements along several different pairs of directions can we establish the presence of quantum correlations. The requirement of measurements in different bases is in fact the difficulty when dealing with modes 
of massive particles. We can measure the number of particles in a mode, but we cannot measure superpositions of particle numbers, as will be explained below [7, 8, 9].

In this paper, we focus on the case of entanglement between spatially separated modes of massive particles. Since, as we have just mentioned, direct measurements on the modes cannot be used to observe nonlocal quantum effects, we ask the question of whether this type of entanglement can be converted into a different type such that nonlocal quantum effects are observable. In spite of the fundamental difficulties associated with particle conservation, some recent studies [10] have suggested that this conversion is in fact possible. Here we analyze several possible methods to observe nonlocal quantum correlations between spatially separated modes without violating any fundamental superselection rules. In particular, we find the rather surprising result that a finite auxiliary Bose-Einstein condensate (BEC) can be used to convert this single-particle entanglement an arbitrary number of times, neglecting difficulties that could arise in a realistic setup.

In fact, this type of single-particle entanglement has attracted significant interest in recent years 11, 12, 13, 14, 15, 16, 17, 18. There seems to be a consensus over the entanglement in a single photon, as will be explained briefly below. However, our proposals presented in this paper achieve the conversion of entanglement in a single massive particle. Our proposals can also be seen as concrete realizations of the recently proposed concept of bound-entanglement activation [19].

The role of the BEC in this problem can be understood, rather figuratively, as follows: the presence of the single (delocalized, or flying) particle can be 'captured on a quantum film', i.e. recorded in the state of a quantum object, such that no collapse of the wave function occurs during this process. One 'quantum film' is placed on each side of the apparatus, so that one of them will record the presence of the flying particle. However, quantum correlations cannot be observed between these 'quantum films', because the particle still carries the which-path information about its location. The BEC can now be seen, in some sense, as a sea of particles identical to the one carrying the which-path information. If the BEC is prepared in a suitable initial state and the flying particle is properly injected into this sea of identical particles, the whichpath information is lost (i.e. truly erased), and quantum correlations can be observed when we measure (or, if you wish, develop) the 'quantum films'. The perfect sea of particles for discarding the flying particle would be a coherent state with many particles. One should note, however, that true coherent states (without a well-defined total particle number) do not exist in systems of massive particles; coherent states usually provide a useful calculational tool, but they should be used with care when dealing with conceptual questions [20, 21]. This is the reason why one must construct the initial state of the BEC such that it emulates, as much as possible, a coherent state for the purposes of the proposed experiment, while still obeying particle-number conservation laws.

The paper is organized as follows: In Sec. II we present a simple experimental setup where the fundamental questions about the possibility of detecting the entanglement between modes can be seen clearly. In Sec. III we present a quantum-eraser approach to detecting the entanglement, and we discuss why we are not interested in that approach. In Sec. IV we discuss how the entanglement can be converted using auxiliary atoms. Section V contains concluding remarks.

\section{BASIC PROBLEM}

Take a particle-beam-splitter setup, as shown in Fig. 1(a) for the case of a photon beam splitter. A particle passes through the beam splitter, resulting in the quantum state:

$$
|\Psi\rangle=\frac{1}{\sqrt{2}}(|L\rangle+|R\rangle) .
$$

When viewed as a quantum state of the particle, the above state clearly does not seem to contain any entanglement. If one thinks of the modes of the field describing that species of particles, however, one finds that the modes on the left and right sides of the beam splitter are entangled; the state is a quantum superposition of the mode on the left containing one particle and the mode on the right empty as well as the opposite combination. The question now is whether this type of entanglement is simply a book-keeping issue that has no physical implications or it leads to measurable effects associated with entanglement, e.g. violation of the Bell inequalities using local measurements [5]. If the particle is a photon, one immediately finds that by positioning two atoms in their ground states, one on each side of the beam splitter, it is possible to design the system such that the photon will excite the corresponding atom. This process is illustrated in Fig. 1(b), and it results in the quantum state

$$
|\Psi\rangle=\frac{1}{\sqrt{2}}(|e g\rangle+|g e\rangle),
$$

where the symbols $g$ and $e$ stand for ground and excited state, respectively, and the first and second symbols describe the atoms on the left and right, respectively. It is now possible to measure the state of each atom in any desired basis (for example, a combination of unitary operations using classical electromagnetic fields and a measurement in the $\{|g\rangle,|e\rangle\}$ basis will do). One should therefore be able to observe a violation of the Bell inequalities when measuring the states of the two atoms [22].

We now ask whether a similar procedure can be followed in the case of massive particles. A key point to note here is that nature allows us to create and destroy photons. In other words, there is no conservation law for the number of photons, and there are physical processes 
(a)

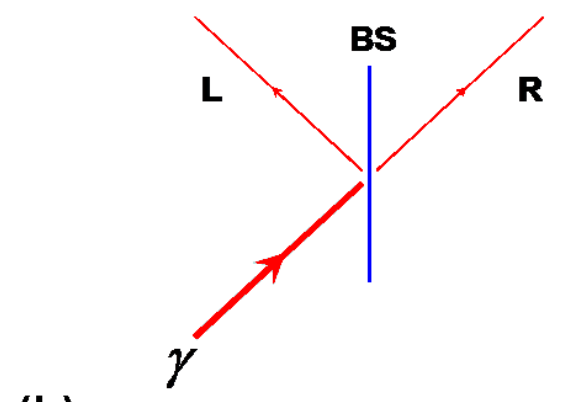

(b)

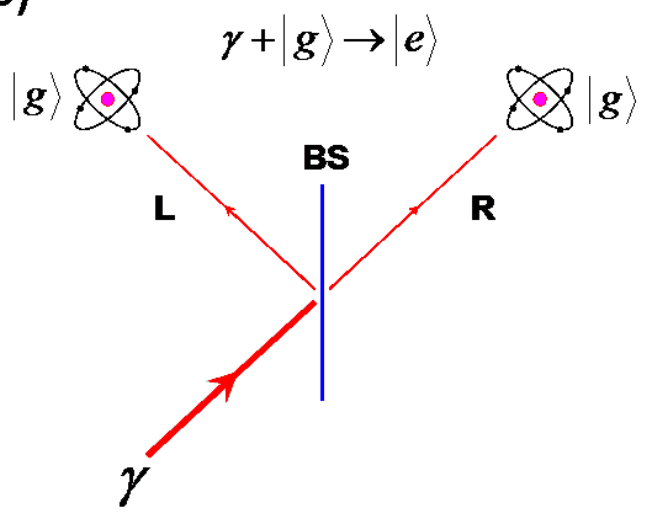

(c)

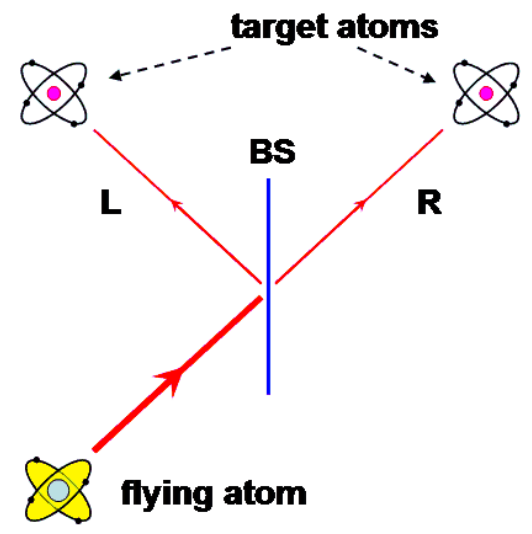

FIG. 1: (color online) Schematic diagrams of beam-splitter setups. Figure 1(a) shows the basic optics setup. Figure 1(b) shows a photon beam-splitter setup with two target atoms, one of which will be excited by absorbing the incident photon. Figure 1(c) shows the atomic analog of Fig 1(b), where the flying atom excites one of the two target atoms.

that change it, e.g. absorption of a photon by an atom. However, there are conservation laws for all massive particles (here we shall not get into high-energy-physics discussions, and we shall pretend, for simplicity, that there is a field describing whatever particle we are considering, e.g. atoms).

We now imagine the situation where a flying atom passes through the beam splitter [23] and ends up in the quantum state of Eq. (11). We take two target atoms as above, and we design the apparatus such that the flying atom interacts with and excites the corresponding target atom, as shown in Fig. 1(c). Instead of the state in Eq. (2), one now finds the state

$$
|\Psi\rangle=\frac{1}{\sqrt{2}}(|L\rangle \otimes|e g\rangle+|R\rangle \otimes|g e\rangle) .
$$

In order to predict the correlations that would be observed in any measurement on the target atoms, one must trace out the flying atom's degree of freedom. One therefore finds the reduced density matrix

$$
\rho=\left(\begin{array}{llll}
0 & 0 & 0 & 0 \\
0 & \frac{1}{2} & 0 & 0 \\
0 & 0 & \frac{1}{2} & 0 \\
0 & 0 & 0 & 0
\end{array}\right),
$$

and the measurement results will be classically correlated. No signature of entanglement can be observed in these results.

From the above example, it is clear that tracing out the degree of freedom of the flying atom prevents us from observing quantum correlations between the target atoms. We can therefore say that the entanglement between the modes in the above setup cannot be tested. In the following sections, we shall try to construct more elaborate setups such that entanglement between the modes of the flying atom can result in measurable entanglement between the target atoms. In the first case, we will use a global measurement on the state of the flying atom. In the other two cases, auxiliary modes of the same species as the flying atom will be used.

\section{QUANTUM-ERASER APPROACH}

We start with perhaps the simplest case. As we discussed above, tracing out the degree of freedom of the flying atom results in classical correlations between the states of the target atoms, but no quantum correlations would be observable. Performing a measurement on the flying atom while it is on one side of the apparatus does not help either, since such a measurement would project the state of the target atoms onto one of two separable state. However, if we perform a measurement on the flying atom that erases the information about which side of the beam splitter it went into, the target atoms would end up in an entangled state. The details are given below.

Let us take the situation where the flying atom was prepared in the superposition state given by Eq. (1), and then excited the corresponding target atom. We therefore have the quantum state of Eq. (3). We now perform a measurement on the flying atom in the basis $\{|+\rangle \equiv(|L\rangle+|R\rangle) / \sqrt{2},|-\rangle \equiv(|L\rangle-|R\rangle) / \sqrt{2}\}$. Depending on the outcome of the measurement, the target atoms end up in the state 


$$
|\Psi\rangle=\frac{1}{\sqrt{2}}(|e g\rangle \pm|g e\rangle),
$$

where the upper and lower signs correspond to the outcomes $|+\rangle$ and $|-\rangle$, respectively. By post-selecting only those instances where the outcome $|+\rangle$ was obtained, quantum correlations would be observable between the target atoms. Naturally one could equally well postselect the instances where the outcome $|-\rangle$ was obtained.

Although the above procedure allows us, in a sense, to observe the entanglement between the modes of the flying atom, it does not meet the criteria we set in Sec. I above: In order to observe the entanglement, we had to perform a global measurement on the state of the flying atom. In other words, after the flying atom excited one of the target atoms, it had to travel (carrying quantum information) to a common detection location, so that its state could be measured in the desired basis. One could therefore argue that the entanglement did not exist in any meaningful form at the time of excitation, but rather the flying atom and measurement apparatus mediated an interaction between the target atoms. Accounting for the time required to establish the measurement results then violates the criteria of Sec. I. We shall therefore not allow such global measurements in the following sections.

\section{OBSERVING ENTANGLEMENT WITHOUT GLOBAL MEASUREMENTS}

As above, we take a flying atom going through a beam splitter and exciting one of two target atoms. Since the flying atom cannot be annihilated by any physical process, this simple setup cannot be used to detect entanglement between the target atoms. We now present two scenarios where auxiliary atoms of the same species as the flying atom can be used to erase, at least partially, the information about which side of the beam splitter the flying atom went into.

\section{A. Scenario I: one auxiliary atom}

Let us imagine that we have already prepared an atom of the same species as the flying atom in the state

$$
\left|\Psi_{\text {aux }}\right\rangle=\frac{1}{\sqrt{2}}\left(\left|L_{\text {aux }}\right\rangle+\left|R_{\text {aux }}\right\rangle\right) .
$$

where the states $\left|L_{\text {aux }}\right\rangle$ and $\left|R_{\text {aux }}\right\rangle$ describe localized modes on the left and right side of the beam splitter, respectively. The quantum state of the entire system can therefore be expressed as

$$
\begin{aligned}
|\Psi\rangle= & \frac{1}{2}\left(\left|L_{\text {aux }}\right\rangle+\left|R_{\text {aux }}\right\rangle\right) \\
& \otimes\left(\left|L_{\text {flying }}\right\rangle \otimes|e g\rangle+\left|R_{\text {flying }}\right\rangle \otimes|g e\rangle\right) .
\end{aligned}
$$

We now wish to use the indistinguishability between the flying atom and the auxiliary atom to erase (even if partially) the which-path information carried by the flying atom. We find it instructive to start with what we consider a non-ideal approach, especially for those who might not be sufficiently familiar with certain details of the procedures discussed below.

\section{Non-ideal approach}

Let us imagine that the flying atom is stopped and trapped after it has excited one of the target atoms (there is no fundamental difficulty with doing that). The flying and auxiliary atoms are now trapped in the ground states of localized potential wells. If we take the quantum state in Eq. (7) and slowly merge the two wells on the left and the two wells on the right, one might expect the resulting state to be given by

$$
|\Psi\rangle=\frac{1}{2}(|2,0\rangle \otimes|e g\rangle+|1,1\rangle \otimes\{|e g\rangle+|g e\rangle\}+|0,2\rangle \otimes|g e\rangle),
$$

where the ket on the left describes the total number of itinerant atoms (i.e. flying and auxiliary) on the left and right sides of the beam splitter. Assuming that the above is true, if we measure the number of atoms in the combined traps, we would have a $50 \%$ chance of finding one atom on each side of the beam splitter. If that happens, we would have lost all information about which side the flying atom went into. We would therefore find the state of the target atoms to be

$$
|\Psi\rangle=\frac{1}{\sqrt{2}}(|e g\rangle+|g e\rangle),
$$

and quantum correlations would be observable in those instances. In the instances where the two atoms (flying and auxiliary) are found on the same side of the beam splitter, we know which side the flying atom went into, and we end up with a separable state for the target atoms.

Although the above scenario might, at first sight, look like it does not violate any principles of quantum mechanics, closer inspection reveals the opposite. In particular, let us take the situation where one atom is on each side of the beam splitter before merging the two pairs of wells. It would be wrong to conclude that the slow merging process results in the state $|1,1\rangle$ (without any additional quantum numbers). If we note that two orthogonal states, namely $\left|L_{\text {aux. }}\right\rangle \otimes\left|R_{\text {flying }}\right\rangle$ and $\left|R_{\text {aux. }}\right\rangle \otimes\left|L_{\text {flying }}\right\rangle$, would result in the same state according to that description, we find that the process of merging the wells must be treated with more care. Let us focus on a pair of wells with one atom in the ground state of one of them. If we take the limit of an infinite barrier height (and zero bias between the two wells), the ground state of the doublewell potential is degenerate (see Fig. 2(a)). When we combine the two wells, the atom will end up in a superposition between the ground and first-excited states of the 
(a)

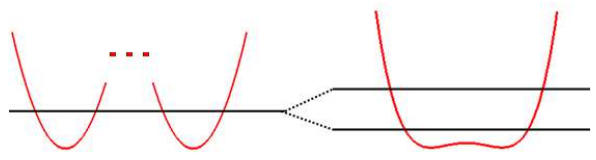

(b)
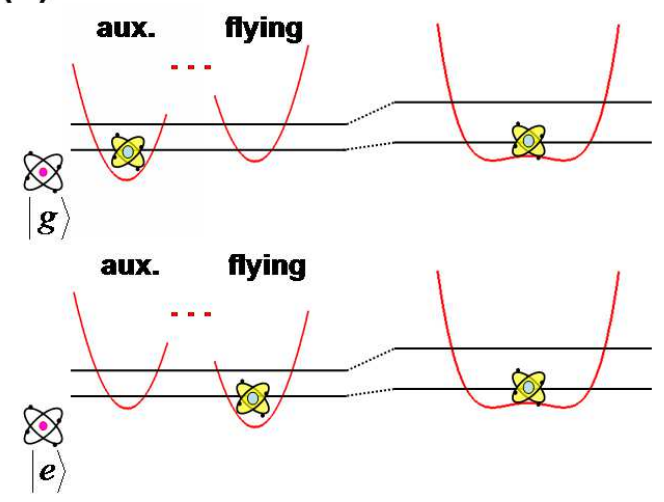

FIG. 2: (color online) Merging two wells into one (note that the two wells being merged are on the same side of the beam splitter). If the initial double-well potential is perfectly symmetric with an infinite barrier (a), the ground state is degenerate, and it splits into two states during the merging process. The symmetry can be lifted using internal-state-dependent interactions with the target atom, ensuring that the atom ends up in the ground state of the merged well (b).

(merged) single well, with amplitudes and a phase factor that depend on the source of the atom (i.e., flying or auxiliary atom) and the details of the merging process. The which-path information is therefore not erased at this stage. It is possible, in principle, to perform certain measurements on the atoms in the merged wells and establish the phase in the entangled state of the target atoms (similarly to what was done in the quantum-eraser approach above). However, since (1) post-selection involving the exchange of information between the two sides would be required in this case and (2) the state resulting from the merging of the wells is strongly susceptible to fluctuations in the bias between the two separate wells, we abandon this approach.

Note that since we only obtain the desired entangled state when the flying atom and auxiliary atom are found on opposite sides of the beam splitter, the difference between bosonic and fermionic particles does not affect our argument. It only affects the instances where both atoms are found on the same side of the beam splitter. But those instances will be discarded. We therefore do not need to analyze the two cases separately.

\section{Better approach}

Let us now take the same procedure as in the 'non-ideal approach' above, except that during the well-merging process we bring the target atoms close to the merging wells. We design the system such that a target atom in the ground state raises the well of the flying atom relative to that of the auxiliary atom, and a target atom in the excited state lowers the well of the flying atom relative to that of the auxiliary atom, as shown in Fig. 2(b). Following this procedure each itinerant atom (assuming one atom was on each side of the beam splitter) will be in the ground state of its potential well. The quantum state is then correctly described by Eq. (8); the desired (partial) erasure of the which-path information has been achieved. We therefore obtain an entangled pair of target atoms $50 \%$ of the time and separable states $50 \%$ of the time. The desired raising and lowering of the potential wells could be achieved using electronic-state-dependent interactions between the itinerant and target atoms.

Three remarks are in order: (1) When merging the two wells, one must avoid back action from the atom on the trapping apparatus. That back action would in effect perform a measurement on the location of the flying atom, preventing the observation of entanglement. Since the trapping apparatus is macroscopic, its recoil from moving the trapped atom should be negligible if designed properly (in particular, one must also avoid resonant scattering in the case of an optical atomic trap). (2) We still discard $50 \%$ of the samples, namely when we find both itinerant atoms on the same side. However, this step does not raise the same concerns that we raised regarding post-selection above, because the decision of discarding a given sample can be made locally, without any exchange of information between the two sides. (3) The two-wellmerging process is essentially equivalent to a standard controlled-NOT gate on two qubits. Before the process, the target atom and the position of the trapped (i.e. flying or auxiliary) atom in the double well are perfectly correlated. Depending on the state of the target atom, i.e. the control bit, we change the position of the trapped atom, such that the final state of the trapped atom is disentangled from that of the target atom. This is exactly the same information erasing process as what was described in Ref. [24] in the context of Maxwell's demon.

\section{Alternative approach: initially correlated flying and auxiliary atoms}

For completeness let us mention the following possibility. If we take the auxiliary atom and the flying atom to go through the beam splitter immediately after each other such that their interaction ensures that each one of them goes to a different side of the beam splitter, we are guaranteed to have the favourable situation with one itinerant atom on each side of the beam splitter. We are thus guaranteed to obtain entanglement between the target atoms. However, the entanglement after crossing the beam splitter in this situation is closer to being an entanglement involving the internal degrees of freedom of two particles than it is to the entanglement between 
modes that we are interested in. In this case, the 'internal' degree of freedom is the time at which the atom goes through the beam splitter: if the atom on the left crossed the beam splitter at the earlier time, the atom on the right must have crossed the beam splitter at the later time, and vice versa. We therefore do not consider this possibility in any more detail.

\section{B. Scenario II: Auxiliary Bose-Einstein condensate}

The above scenario allows us to obtain an entangled pair of target atoms $50 \%$ of the time. Since only one auxiliary atom was used there, it is natural to ask whether replacing the auxiliary atom with an auxiliary BEC would result in a higher probability for producing a pair with the desired entanglement. Furthermore, one could still argue that when using an auxiliary atom one is not probing the entanglement from the flying atom, but rather a complicated form of entanglement involving both the flying and auxiliary atom. These issues will be addressed below.

Before proceeding let us also note here that the conversion, or transfer, of entanglement from the flying atom to the target atoms was made possible by the fact that the auxiliary atom was not localized in the initial state. The quantum fluctuations associated with our lack of knowledge about the number of auxiliary atoms on the left and right sides of the beam splitter allowed us to erase the which-path information carried by the flying atom. After the merging of the wells (and discarding the instances where both flying and auxiliary atoms turned out on the same side of the beam splitter), there is no way, even in principle, to tell whether the flying atom went to the left or right. A natural generalization would therefore be to use a large number of auxiliary atoms where the addition of a single atom would be hardly noticeable. One is therefore led to think of coherent states. As mentioned in Sec. I, however, truly coherent states cannot be prepared in this system. The closest one can get to them is a single BEC (with a well-defined number of atoms) shared between the two sides of the beam splitter, such that it is allowed to have quantum fluctuations in the number of atoms on each side.

We now present a procedure based on the abovementioned idea. We take a BEC of non-interacting atoms trapped in a single well, and we split the well into two and take those to opposite sides of the beam splitter. The state of the BEC is given by

$$
\begin{aligned}
\left|\Psi_{\mathrm{BEC}}\right\rangle & =\frac{1}{\sqrt{N !}}\left(\frac{a_{L, \mathrm{aux}}^{\dagger}+a_{R, \text { aux }}^{\dagger}}{\sqrt{2}}\right)^{N}|\mathrm{vac}\rangle \\
& =\sum_{j=0}^{N} \sqrt{f_{N, j}}|j, N-j\rangle_{\mathrm{aux}}
\end{aligned}
$$

where the operators $a_{L, \text { aux }}^{\dagger}$ and $a_{R \text {,aux }}^{\dagger}$ create atoms of the same species as the flying atom in auxiliary modes on the left and right sides of the beam splitter, respectively, $N$ is the total number of atoms in the condensate, and $|\mathrm{vac}\rangle$ is the vacuum state for that species [25, 26, 27]. The function $f_{N, j}$ is defined as

$$
f_{N, j} \equiv \frac{1}{2^{N}} \times \frac{N !}{j !(N-j) !} .
$$

In the ket of the second line of Eq. (10), the first and second numbers denote the number of atoms in the BEC on the left and right sides of the beam splitter, respectively (note that the total number of atoms in the entire system is well defined, in accordance with superselection rules; the number of atoms in the left and right wells, however, is not well defined). The state of the entire system is therefore given by

$$
\begin{aligned}
|\Psi\rangle= & \sum_{j=0}^{N} \sqrt{f_{N, j}}|j, N-j\rangle_{\text {aux }} \\
& \otimes \frac{1}{\sqrt{2}}\left(\left|L_{\text {flying }}\right\rangle \otimes|e g\rangle+\left|R_{\text {flying }}\right\rangle \otimes|g e\rangle\right) .
\end{aligned}
$$

We now follow a similar approach to the auxiliary-atom scenario of Sec. IVA2 and slowly merge the trapping well of the BEC with that of the flying atom [28]. We can now express the state of the system as

$$
\begin{aligned}
|\Psi\rangle=\frac{1}{\sqrt{2}} & \left(\frac{1}{2^{N / 2}}|N+1,0\rangle \otimes|e g\rangle+\frac{1}{2^{N / 2}}|0, N+1\rangle \otimes|g e\rangle\right. \\
+ & \sum_{j=1}^{N}|j, N+1-j\rangle \\
& \left.\otimes\left\{\sqrt{f_{N, j-1}}|e g\rangle+\sqrt{f_{N, j}}|g e\rangle\right\}\right)
\end{aligned}
$$

By tracing over the BEC degree of freedom, we find that the relevant off-diagonal element of the target atoms' reduced density matrix is given by

$$
\begin{aligned}
\rho_{g e, e g} & =\frac{1}{2} \sum_{j=1}^{N} \sqrt{f_{N, j-1}} \times \sqrt{f_{N, j}} \\
& =\frac{1}{2} \sum_{\delta j=-\left(\frac{N}{2}-1\right)}^{\frac{N}{2}} f_{N, \frac{N}{2}+\delta j} \times \sqrt{\frac{\frac{N}{2}+\delta j}{\frac{N}{2}-\delta j+1}} \\
& \approx \frac{1}{2} \times\left(1-\frac{1}{2 N}\right),
\end{aligned}
$$

where we have defined $\delta j \equiv j-N / 2$ and assumed that $N$ is even in the intermediate steps. Combining the above expression for $\rho_{g e, e g}$ with the fact that $\rho_{g e, g e}=\rho_{e g, e g}=$ $1 / 2$, the probability of successfully generating the desired entangled state is found to approach unity, with an error that decreases as $1 / N$. To put it differently, the concurrence $C$, which can be calculated straightforwardly [29], is given by 


$$
C=1-\frac{1}{2 N}
$$

The above expression for the concurrence turns out to be a good approximation even for small values of $N$, with accidental agreement for $N=1$.

It would seem wasteful to use a large condensate to generate a single pair of entangled target atoms. The next question to ask is therefore how many times we can use the same condensate to generate entangled pairs of target atoms from a stream of flying atoms. In order to answer this question, we take the quantum state in Eq. (13) and trace out the target atoms' degrees of freedom. We find that the BEC reduced density matrix is given by

$$
\rho_{\mathrm{BEC}}^{\text {after } 1 \text { st run }}=\frac{1}{2}\left(\left|\Lambda_{L}\right\rangle\left\langle\Lambda_{L}|+| \Lambda_{R}\right\rangle\left\langle\Lambda_{R}\right|\right),
$$

where

$$
\begin{aligned}
& \left|\Lambda_{L}\right\rangle=\sum_{j=0}^{N} \sqrt{f_{N, j}}|j+1, N-j\rangle \\
& \left|\Lambda_{R}\right\rangle=\sum_{j=0}^{N} \sqrt{f_{N, j}}|j, N+1-j\rangle .
\end{aligned}
$$

The subscript of $\Lambda$, i.e. $\mathrm{L}$ or $\mathrm{R}$, indicates the path that the first flying atom took, which is also copied to the state of the target atoms. The density matrix in Eq. (16) describes a mixed state, which might suggest that the probability of successful production of a second entangled pair of target atoms will be reduced. We note, however, that each of the two pure states $\left|\Lambda_{L}\right\rangle$ and $\left|\Lambda_{R}\right\rangle$ has exactly the same atom-number distribution as the original BEC state, except that the center of the distribution can be shifted. If we follow the same procedure as above with the BEC in this new mixed state, the result can be obtained by taking the average of the expected results from states $\left|\Lambda_{L}\right\rangle$ and $\left|\Lambda_{R}\right\rangle$. Since each one of those gives identical results to the case of a pure BEC state with $N$ atoms, we find that the second flying atom produces an entangled state between the target atoms with the same error (of order $1 / N$ ) as the first flying atom. The procedure can be repeated indefinitely, with the error remaining at $1 /(2 N)$, where $N$ is the initial number of atoms in the condensate. It is quite remarkable that we obtain this result even when the procedure has been repeated more than $N$ times and the (classical) fluctuations in the number of atoms on the left and right sides exceed the natural (quantum) fluctuations, of order $\sqrt{N}$, that were present in the original BEC. In practice, the process cannot be repeated infinitely many times, simply because the model of non-interacting particles would break down at some point. However, this limitation has nothing to do with the effect that we are considering, and the system can be designed such that it only appears after a number of repetitions that is much larger than $N$.

It is worth mentioning two remarks regarding the procedure involving the stream of flying atoms. (1) When we traced over the target atoms' degrees of freedom, we have excluded knowledge about the BEC density matrix that we would have obtained by keeping a record of the outcome of measurements on those atoms. However, since we are looking for correlations between the states of the target atoms only, and we are not interested in correlations between those and the BEC, it is safe to trace over their degrees of freedom when considering subsequent repetitions of the procedure. (2) It is natural to think of the BEC as a catalyst for converting the entanglement, rather than contributing its own entanglement to the resulting pairs of target atoms. That can be seen by observing the fact that the BEC can be reused in the procedure indefinitely.

\section{CONCLUSION}

We have discussed the fundamental difficulty with directly observing nonlocal quantum effects, e.g. testing Bell inequalities, using the entanglement between spatially separated modes of massive particles. These difficulties apply to some basic procedures attempting to convert this mode entanglement into Bell-testable forms. We have then analyzed several possible approaches where this conversion can be achieved by using auxiliary particles, including the case of an auxiliary BEC. Remarkably we found that in the case of a BEC, the number of times we can convert such entanglement is not limited by the number of particles in the BEC. Although we have not given mathematical proofs, we suspect that the procedures we propose provide the maximum obtainable conversion probabilities using a given number of particles. Our results using a single auxiliary atom apply regardless of whether the particles are bosons or fermions. However, we cannot see any simple analogue of the BEC method in the case of fermionic flying atoms.

\section{Acknowledgments}

We would like to thank T. Iitaka, A. Lvovsky, D. Markham and A. Zavatta for useful discussions. This work was supported in part by the National Security Agency (NSA), the Laboratory for Physical Sciences (LPS) and the Army Research Office (ARO); and also by the National Science Foundation (NSF) grant No. EIA0130383. One of us (S.A.) was supported by the Japan Society for the Promotion of Science (JSPS). 
[2] A. Einstein, B. Podolsky, and N. Rosen, Phys. Rev. 47, 777 (1935); N. Bohr, Phys. Rev. 48, 696 (1935).

[3] See, e.g., M. A. Nielsen and I. L. Chuang, Quantum Computation and Quantum Information (Cambridge University Press, 2000); J. Stolze and D. Suter, Quantum Computing: A Short Course from Theory to Experiment (Wiley-VCH, Weinheim, 2004).

[4] For example, a spin-3/2 particle can be described as two spin-1/2 particles. See, e.g., Y. Hirayama, A. Miranowicz, T. Ota, G. Yusa, K. Muraki, S. K. Ozdemir, and N. Imoto, J. Phys.: Cond. Mat. 18, S885 (2006).

[5] See, e.g., J. S. Bell, Rev. Mod. Phys. 38, 447 (1966).

[6] The exchange of classical information required in order to establish the presence of quantum correlations is not included here. That step will naturally take longer than $d / c$.

[7] G. C. Wick, A. S. Wightman, and E. P. Wigner, Phys. Rev. 88, 101 (1952).

[8] See, e.g., F. Verstraete and J. I. Cirac, Phys. Rev. Lett. 91, 010404 (2003).

[9] Here we have in mind local measurements. We therefore do not include the possibility of measuring superpositions of different distributions of a well-defined number of particles between multiple, spatially separated modes [26].

[10] M. O. Terra Cunha, J. A. Dunningham, and V. Vedral, quant-ph/0606149

[11] S. M. Tan, D. F. Walls, and M. J. Collett, Phys. Rev. Lett. 66, 252 (1991).

[12] L. Hardy, Phys. Rev. Lett. 73, 2279 (1994).

[13] H. M. Wiseman and J. A. Vaccaro, Phys. Rev. Lett. 91, 097902 (2003).

[14] B. Hessmo, P. Usachev, H. Heydari, and G. Björk, Phys. Rev. Lett. 92, 180401 (2004).

[15] S. A. Babichev, J. Appel, and A. I. Lvovsky, Phys. Rev. Lett. 92, 193601 (2004).

[16] S. J. van Enk, Phys. Rev. A 72, 064306 (2005).

[17] S. D. Bartlett, A. C. Doherty, R. W. Spekkens, and H. M. Wiseman, Phys. Rev. A 73, 022311 (2006).

[18] M. D'Angelo, A. Zavatta, V. Parigi, and M. Bellini, Phys. Rev. A 74, 052114 (2006); J. Mod. Opt. 53, 2259 (2006).

[19] M. Horodecki, P. Horodecki, and R. Horodecki, Phys. Rev. Lett. 80, 5239 (1998); P. Horodecki, M. Horodecki, and R. Horodecki, Phys. Rev. Lett. 82, 1056 (1999); see also Ref. [17].
[20] See, e.g., Sec. III.D.1 of Ref. [26].

[21] Examples of results that cannot be obtained using the coherent-state approach can be found in, e.g., A. B. Kuklov and B. V. Svistunov, Phys. Rev. Lett. 89, 170403 (2002); S. Ashhab and A. J. Leggett, Phys. Rev. A 68, 063612 (2003); E. J. Mueller, T.-L. Ho, M. Ueda, and G. Baym, Phys. Rev. A 74, 033612 (2006).

[22] In actual experiments, the violation of Bell inequalities using single photons was observed using direct measurements on the photons. The methods used there require knowledge of quantum-optics techniques. We shall therefore not explain them here [15, 18].

[23] For concrete possible implementations of this process and others discussed in this paper, see e.g. Refs. 30, 31, 32]. We stress, though, that we are mainly interested in fundamental questions here, and one can think of the experiments we propose here as thought experiments. We only mention certain experimental procedures in order to demonstrate that there is no fundamental objection to the processes we discuss.

[24] K. Maruyama, C. Brukner, and V. Vedral, J. Phys. A: Math. Gen. 38, 7175 (2005).

[25] M. R. Andrews, C. G. Townsend, H.-J. Miesner, D. S. Durfee, D. M. Kurn, and W. Ketterle, Science 275, 637 (1997).

[26] See, e.g., A. J. Leggett, Rev. Mod. Phys. 73, 307 (2001).

[27] A. P. Hines, R. H. McKenzie, and G. J. Milburn, Phys. Rev. A 67, 013609 (2003).

[28] Here we need that a target atom in the ground state favours zero occupation of the flying atom trap, whereas a target atom in the excited state favours single-particle occupation of the trap. This situation could in principle be achieved using a combination of target-atommediated interactions between the flying-atom species and trapping-well-dependent interaction resonances.

[29] W. K. Wootters, Phys. Rev. Lett. 80, 2245 (1998).

[30] M. Zhang, P. Zhang, M. S. Chapman, and L. You, Phys. Rev. Lett. 97, 070403 (2006).

[31] T. Calarco, U. Dorner, P. S. Julienne, C. Williams, and P. Zoller, Phys. Rev. A 70, 012306 (2004).

[32] A. M. Dudarev, R. B. Diener, B. Wu, M. G. Raizen, and Q. Niu, Phys. Rev. Lett. 91, 010402 (2003). 